\title{
Diagnostically Oriented Magnetomechanical Effects Analysis in Quasi Static Low Magnetic Field
}

\begin{abstract}
P. Szulim* AND S. Gontarz
Institute of Vehicles, Warsaw University of Technology, L. Narbutta 84, 02-524 Warsaw, Poland

The aim of the work is to try to separate two informations related to the magnetization of the material and the change in its magnetic permeability during the tensile test. These two parameters, in accordance with the adopted model, are associated with reversible and irreversible magnetomechanical effects present in the material degradation process. For extracting the material features, stimulation was performed using a slow-changing magnetic field with a value close to the natural field of the earth. For the observation field on the sample surface an original matrix of triaxial magnetic field sensors was used. Several measuring series were made for various construction steels for various load stages, both for the area of elastic and plastic deformation. The proposed approach together with the results from the conducted research allowed for a better identification of the magnetomechanical effects that carry diagnostic information about the material.
\end{abstract}

DOI: 10.12693/APhysPolA.137.703

PACS/topics: diagnostics, stress, magnetic flux

\section{Introduction}

In contemporary diagnostics non-destructive testing plays increasingly important role in diagnosis of state of technical objects. While referring to the current state of knowledge it is worth stressing that in the case of steel structures, concrete structures, as well as machines, attention is now mainly focused on detecting faults and defects in the earliest stage of their development. Together with technological development of magnetic sensors and measuring instruments, the approach makes the recently discovered magnetic methods in technical diagnostics very attractive. Additionally, bearing in mind the consequences of unpredictable construction disasters and technical object breakdowns, it becomes necessary to advance the science in the direction of technology to be able to detect early phases of fault development. This is possible thanks to use of magnetic methods which rely on the characteristic magnetic and magnetic domain behavior [1-3]. Three significant problems were encountered at the stage of work on the use of a magnetic field registered around the tested object in order to determine its technical condition: selecting the appropriate diagnostic information hidden in the magnetic field signal, separating the various co-existing effects affecting the resulting field distribution, and the appropriate presentation of results. In papers [4-7], the first attempts to separate various effects were carried out. Successes and difficulties in the practical use of the methods proposed there, prompted the authors to carry out an experiment allowing observation of changes of diagnostically interesting parameters with simultaneous controlled mechanical loading of the tested sample.

*corresponding author; e-mail: przemyslaw.szulim@pw.edu.pl
This approach allows the development of a method that separates diagnostic information observed in a magnetic field. It is known that many mechanical phenomena associated with aging, fatigue, or the state of strain of the material lead to some changes in the magnetic properties of steel. These changes can be further observed in a magnetic field close to the material. The difficulty lies in the fact that many of the magnetomechanical phenomena occurs simultaneously, so it is difficult to distinguish the effects of the reasons that cause them [8, 9]. The paper describes a method that allows distinguishing two basic effects.

\section{Mathematical background}

The idea of the separation of measurement quantities is based on the formulation of a measurement model that takes into account the magnetic field components and phenomena affecting the measurement [6]. Since the rotation of the sample is difficult to carry out under actual conditions, it has been proposed to stimulate the sample with an artificial field through the yoke. Sinusoidal excitation changes were assumed. Due to the low value of the generated flux and the current control in the excitation circuit, this assumption could be met. In this case, the local magnetic field value (1) on the surface of the sample can be described using three equations of simple form

$$
B_{M i}=S_{i} \sin \left(\omega t+\varphi_{i}\right)+B_{C i},
$$

where $i$ indicates $x, y$, or $z$ direction of investigated sample.

Again, the $S$ factor (no unit) is related to the magnetic permeability of the sample and describes the degree of magnetic field excitation on the surface of the sample. When $S=0$, this means that the entire excitation flow is enclosed in the sample volume and does not leak outside. The coefficient $\varphi_{i}$ is related to the presence 


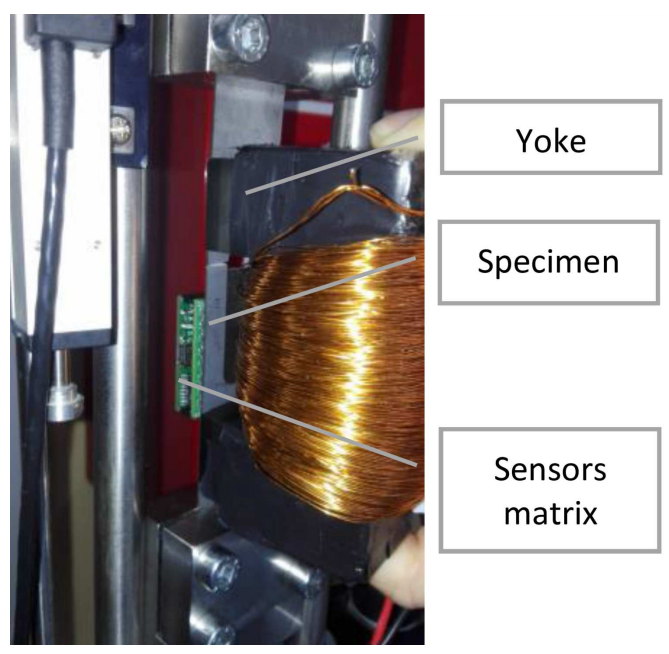

Fig. 1. The key elements of the measuring stand.

of a phase shift between force and the registered field, while the component $B_{C i}$ represents the total effect of the magnetic field of the sample and the surrounding field. By registering the $B_{M i}$ field for full periods of stimulation, one can estimate the desired equation coefficients and treat them as parameters carrying information about the processes occurring in the material under study.

\section{Test stand description}

In the field measurement experiment, developed measuring devices were used (Fig. 1 shows MagMouse). It consists of a matrix of three-axis magnetoresistance sensors, an optical displacement sensor (not used in experiments), and a microcontroller providing communication with an external data recording application. Honey well magnetic sensors with the designation HMC5983 were used for the construction of the device, where the measuring element was the AMR magnetoresistor. The test sample was placed in a testing machine. A matrix and a yoke forcing a controlled magnetic flux were applied to the sample. The level of yoke force was selected experimentally so that the range of field changes was close to the range of the natural field observed on the Earth. A controlled current source was used to set the current in the yoke.

The acquisition process and the generation of signals was managed by an application written in the LabView environment. Signals such as yoke current, tensile force, sample elongation, and magnetic fields were recorded. The experiment was used to test samples made of steel: $4 \mathrm{H} 13$ (1.4034). Each experiment consisted of 24 measurements. At each step, a specific value of the loading force was set and then a magnetic flux was generated and measurement signals were recorded for four periods of the forcing signal. The frequency of the forcing signal was constant and was $0.05 \mathrm{~Hz}$. The frequency of magnetic field sampling was limited by the capabilities of the magnetic field sensors used and was $75 \mathrm{~Hz}$.

\section{Experimental study}

The interesting results obtained in the first experiment induced the authors to study samples for various effort states. The most important results are presented in Fig. 2. It shows the view of the location of sensors in direct contact with the test sample. There are four rows of sensors here. As a result of the experiment, the sample was broken and the interruption occurred at around 20 and 21 sensor number. Figure 3 shows the strength characteristics for the selected experiment and the sample. A range of elastic and plastic deformations is disclosed. The designation of characteristic points has been introduced, which will be subject to a more thorough analysis. Figure 4 shows the course of changes in the constant value of the magnetic field registered by the sensor No. 24 .

The location corresponds to half of the length of the right sample arm (Fig. 2). In accordance with Fig. 3, the range of elastic loads corresponds to measurements 1-9. In this area, the magnetic field components undergo the most significant changes, while in the field of plastic deformations, the changes are relatively small. The peak of change is for stresses around $170 \mathrm{MPa}$, which is half of the elastic range for the specimen material.

Figure 5 shows changes in the waveforms of $S$ parameters in three directions. The highest values occur in the $y$ direction, which is consistent with the expectation,

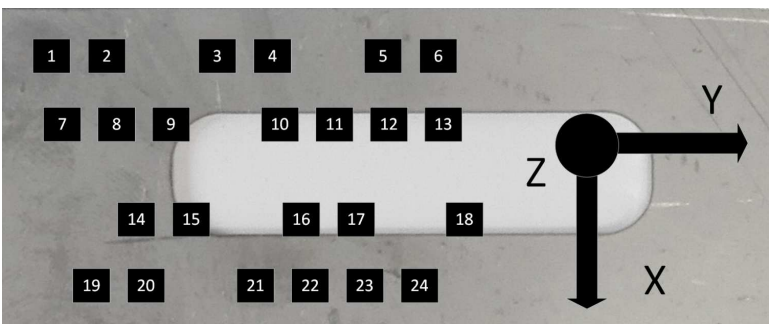

Fig. 2. A fragment of the tested sample with an indication of the locations and numbers of each measurement sensors.

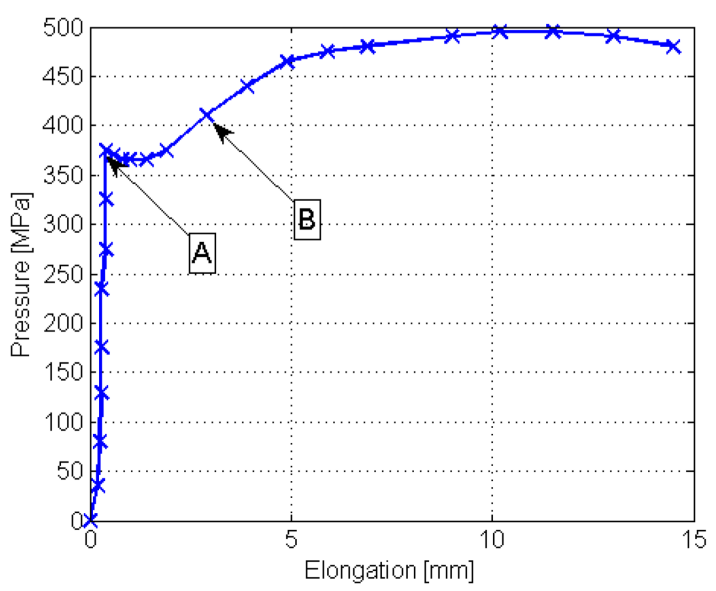

Fig. 3. Strength characteristics for the tested sample. 


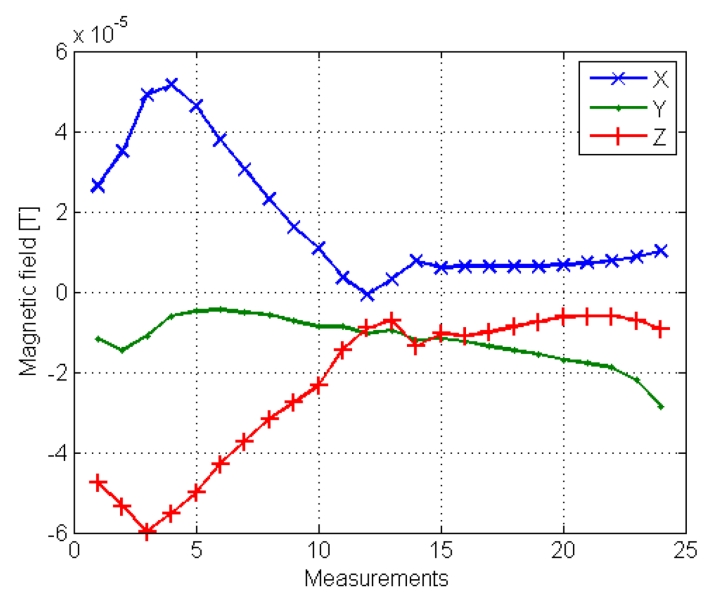

Fig. 4. Magnetic field for sensor No. 24 for subsequent measurements.

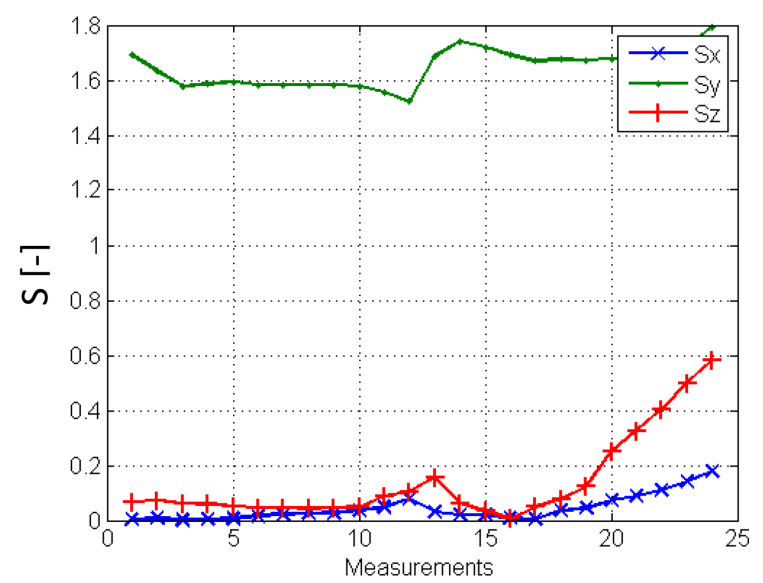

Fig. 5. Estimates of $S$ coefficients for subsequent measurements for sensor No. 24.

because it is the main direction of the magnetic flux generated by the yoke. In the area of measurement 13-15, a significant stroke is revealed. It falls in the vicinity of point $\mathrm{B}$ of strength characteristics (Fig. 3), i.e., in the field of plastic deformation. The measurement at point A (measurement 9) was taken as the limit of elasticity. It also reveals a linear range of changes in the values of these parameters for deeper plasticizing of the material. Figures 6 and 7 show maps representing the field distribution along one set of sensors (19-24) for subsequent measurements.

Figure 7, like Fig. 4, shows that the largest spatial changes in the field distribution occur for the elastic area (measurements 1-9). In the field of plastic, these changes are also visible but they are smaller. The sample was interrupted in the sensor area 20 and 21, where there is a field close to 0 . In Fig. 6 it can be seen that the polarity of the magnetic pole around the fracture area increases even in the area of plastic deformation. However, field change values for the $y$ axis are more than twice smaller than for the $z$ (normal) magnetic field

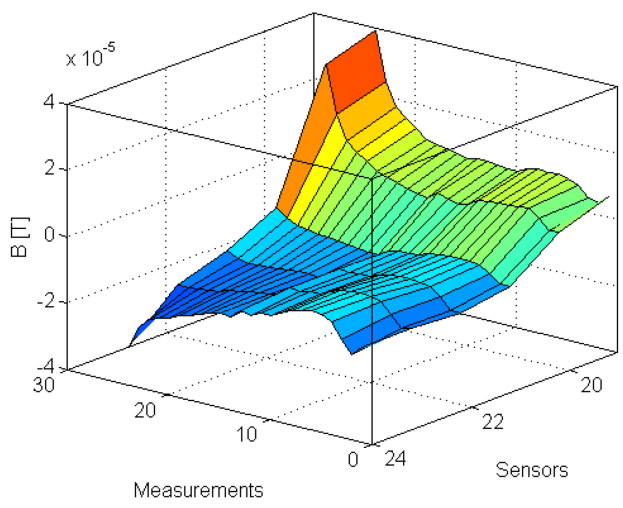

Fig. 6. Field distribution ( $y$ component) along the sample arm for subsequent measurements.

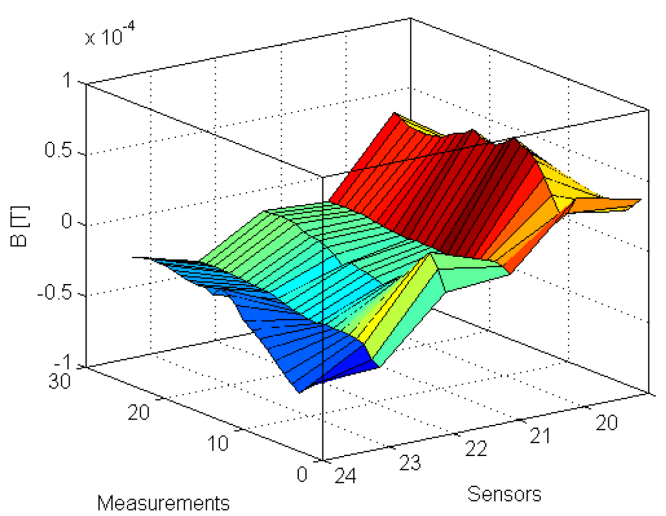

Fig. 7. Field distribution ( $z$ component) along the sample arm for subsequent measurements.

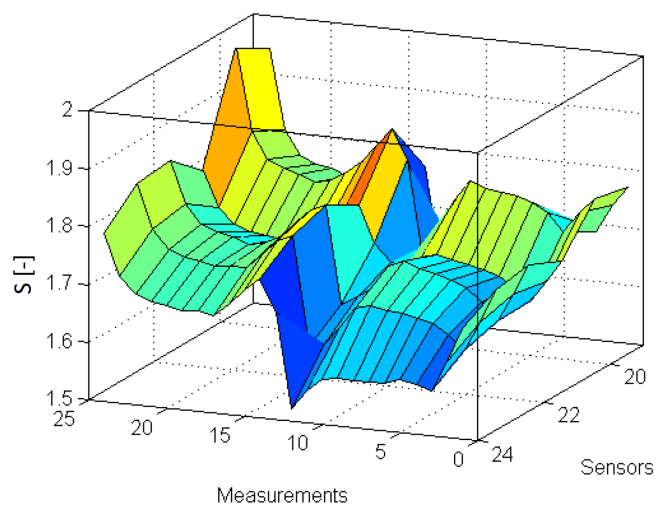

Fig. 8. Distribution of the $S$ parameter in $y$ direction along the sample arm for subsequent measurements.

component. Equally interesting is the course of the $S$ parameter changes describing the scattering of the induced field around the sample. Similar to the single waveform shown in Fig. 3, for the remaining measurements on the specimen arm, a characteristic flat section of the graph for elastic deformations is revealed (Figs. 8, 9). 


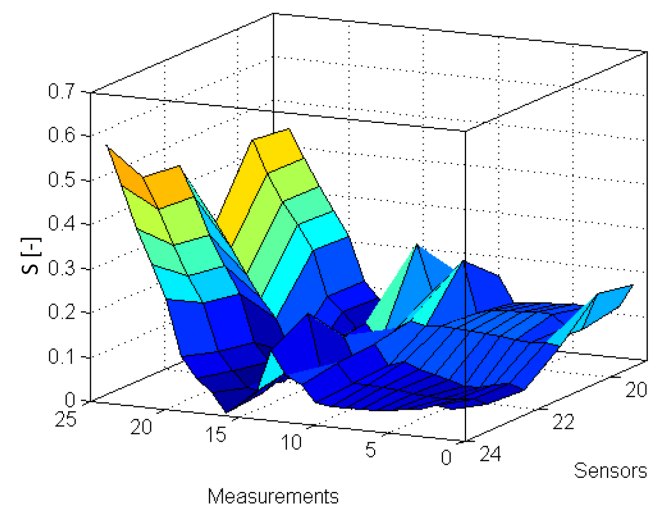

Fig. 9. Distribution of the $S$ parameter in $z$ direction along the sample arm for subsequent measurements.

Then, after crossing the yield point, in the vicinity of measurements $13-15$ there is a characteristic jump and a significant increase in the field dispersion visible especially in the normal direction $(z)$, as shown in Fig. 9. Similar results were observed for the other samples tested.

\section{Conclusions}

As expected, the tests have shown that the area of stress concentration causes local magnetization of the material, which can be used to identify the state of stress of the material in the elastic range. In the plastic state range, spatial fluctuations of the field are much smaller. Research has also shown that stimulation with an external weak field does not cause this phenomena. On the other hand, in the field of plastic deformation, changes in the field spreading parameter are revealed, which are much smaller in the elastic area. Observation of both these parameters could be used to more reliable assessment of the material stress. Phenomena that cause a characteristic jump occurring in point $B$ of the material strength curve will be the subject of further research.

\section{References}

[1] Electromagnetic nondestructive evaluation (XVII), Eds. K. Capova, L. Udpa, L. Janousek, B.P.C. Rao, IOS Press, Amsterdam 2014.

[2] J. Blitz, Electrical and Magnetic Methods of Nondestructive Testing, Adam Hilger, Bristol 1991.

[3] D. Jackiewicz, R. Szewczyk, J. Salach, A. Bieńkowski, Acta Phys. Pol. A 126, 392 (2014).

[4] S. Gontarz, P. Szulim, Y. Lei, J. Magn. Magn. Mater. 467, 29 (2018).

[5] S. Gontarz, P. Szulim, Environm. Eng. Manag. J. 15, 491 (2016).

[6] S. Gontarz, J. Maczak, P. Szulim, Online Monitoring of Steel Constructions Using Passive Methods WCEAM, 2015, p. 625.

[7] P. Szulim, S. Gontarz, J. Electr. Eng. 66, 193 (2015).

[8] P. Szulim, S. Gontarz, J. Maczak, J. Electr. Eng. 66, 203 (2015).

[9] S. Gontarz, S. Radkowski, IEEE Trans. Magn. 48 1143 (2012). 